\title{
Acute effects of anterior thigh foam rolling on hip angle, knee angle, and rectus femoris length in the modified Thomas test
}

Andrew D Vigotsky, Gregory J Lehman, Bret Contreras, Chris Beardsley, Bryan Chung, Erin H Feser

Background: Foam rolling has been shown to acutely increase range of motion (ROM) during knee flexion and hip flexion with the experimenter applying an external force, yet no study to date has measured hip extensibility as a result of foam rolling with controlled knee flexion and hip extension moments. The purpose of this study was to investigate the acute effects of foam rolling on hip extension, knee flexion, and rectus femoris length during the modified Thomas test.

Methods: Twenty-three healthy participants (male $=7$; female $=16$; age $=22 \pm 3.3$ years; height $=170 \pm 9.18 \mathrm{~cm}$; mass $=67.7 \pm 14.9 \mathrm{~kg}$ ) performed two, one-minute bouts of foam rolling applied to the anterior thigh. Hip extension and knee flexion were measured via motion capture before and after the foam rolling intervention, from which rectus femoris length was calculated.

Results: Although the increase in hip extension (change $=+1.860(+0.11,+3.61) ; z(22)=$ 2.08; $p=0.0372 ;$ Pearson's $r=0.43(0.02,0.72))$ was not due to chance alone, it cannot be said that the observed changes in knee flexion (change $=-1.390(-5.53,+2.75) ; \mathrm{t}(22)=$ $-0.70 ; p=0.4933$; Cohen's $d=-0.15(-0.58,0.29)$ ) or rectus femoris length (change $=$ $0.005(-0.013,+0.003) ; \mathrm{t}(22)=-1.30 ; p=0.2070$; Cohen's $d=-0.27(-0.70,0.16))$ were not due to chance alone.

Conclusions: Although a small change in hip extension was observed, no changes in knee flexion or rectus femoris length were observed. From these data, it appears unlikely that foam rolling applied to the anterior thigh will improve hip extension and knee flexion ROM, especially if performed in combination with a dynamic stretching protocol. 


\title{
Acute effects of anterior thigh foam rolling on hip angle, knee angle, and rectus femoris
}

\author{
length in the modified Thomas test
}

Running Head: FOAM ROLL

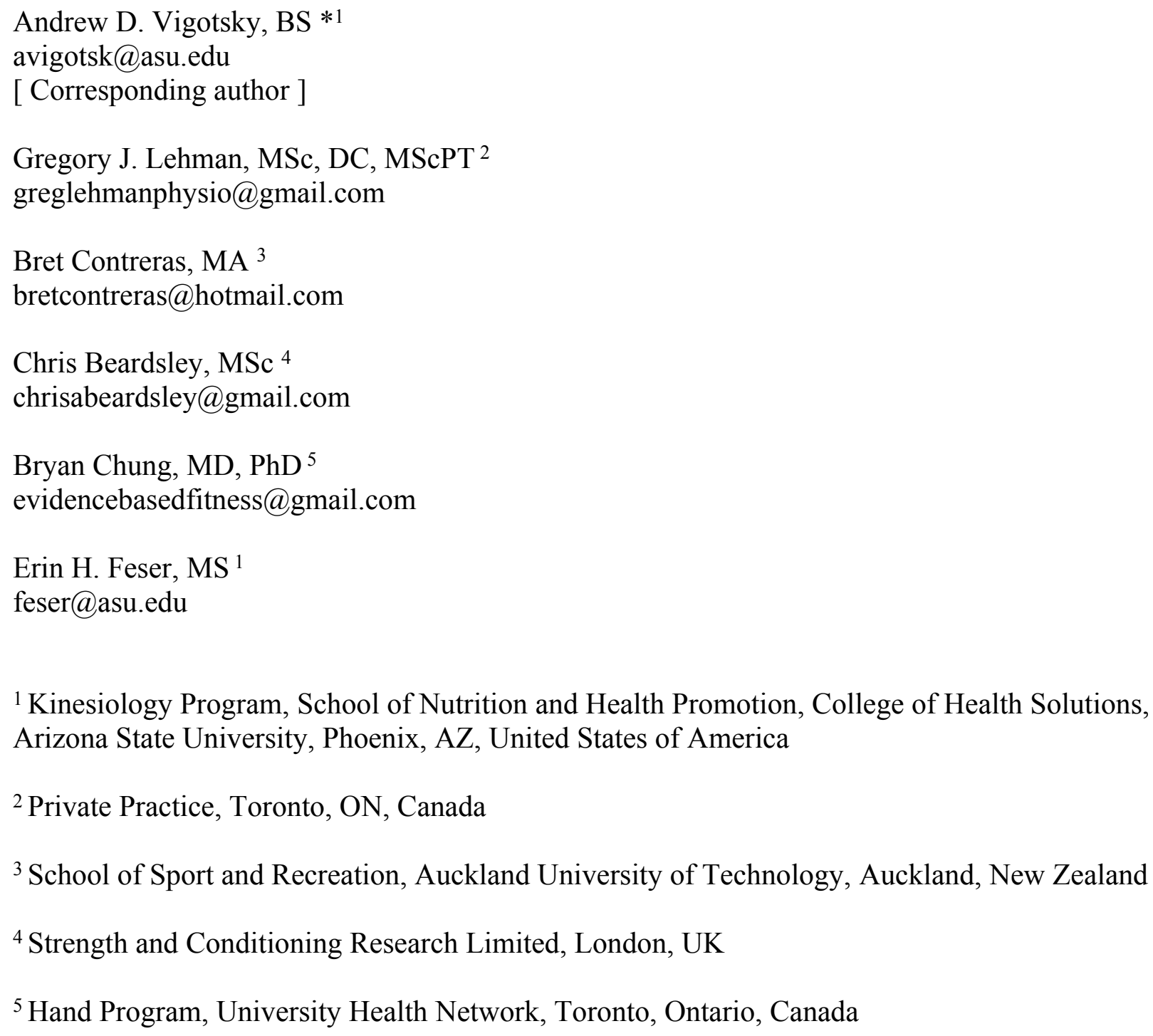




\section{Introduction}

Foam rolling (FR) is a ubiquitous intervention, performed by athletes during both preparation for and following physical activity. FR is postulated to be a form of self-myofascial release, despite no research investigating whether FR directly influences fascia. Therefore, it is perhaps presumptuous to refer to the fascia in its name, let alone as its mediator. For the purposes of this study, FR and similar modalities, such as massage sticks, will be referred to as self-manual therapy. Previous self-manual therapy and FR work has been shown to increase range of motion (ROM) (Behara \& Jacobson 2015; Halperin et al. 2014; Jay et al. 2014; MacDonald et al. 2013; Schroeder \& Best 2015; Škarabot et al. 2015; Sullivan et al. 2013), attenuate delayed onset muscle soreness (DOMS) (Macdonald et al. 2014; Pearcey et al. 2014), reduce arterial stiffness (Okamoto et al. 2013), and improve vascular endothelial function (Okamoto et al. 2013). Importantly, FR has also been shown not to have detrimental effects on physical performance (Behara \& Jacobson 2015; Halperin et al. 2014; Healey et al. 2013; MacDonald et al. 2013; Sullivan et al. 2013).

Quadriceps injuries are a commonplace in sport (Orchard \& Seward 2002), and the rectus femoris is the most commonly injured quadriceps muscle (Cross et al. 2003; Speer et al. 1992). Risk factors for injury appear to be multifactorial (Mendiguchia et al. 2012). Of particular interest are hip flexor strength and flexibility, as these can be modified through training and warm-up (Mendiguchia et al. 2012). Should FR be an efficacious methodology for increasing rectus femoris extensibility, it may allow athletes to train through a greater ROM. Training through a greater ROM would allow athletes to not only see greater gains in strength throughout a greater ROM, but also increase their total ROM (Hartmann et al. 2012; McMahon et al. 2014; 
Morton et al. 2011; Wyon et al. 2013). Because both strength and flexibility are risk factors for

60

\section{Methods}

The effectiveness of self-manual therapy in increasing ROM is of particular interest, as flexibility appears to be a risk factor for muscle strain injury (Mendiguchia et al. 2012). As noted by Schroeder \& Best (2015), despite the heterogeneity of previous studies, FR does appear to be an efficacious intervention for increasing flexibility. Only a couple of studies have investigated the effects of self-manual therapy on hip flexor - namely, rectus femoris - extensibility, as measured by either knee flexion or hip extension. The first to do so was MacDonald et al. (2013), who found that two, one-minute bouts of FR applied to the anterior thigh increased knee flexion ROM by $10^{\circ}$ and $8^{\circ}$ at 2 and 10 minutes post-intervention, respectively. More recently, Bushell et al. (2015) investigated the effects of three, one-minute bouts of FR applied to the anterior thigh on hip extension angle during a dynamic lunge. The intervention was completed once per week for three weeks. The only increase in hip extension noted was during the second week; no changes were found in the first or third weeks. Investigators did report a slight increase in hip extension from the first to second week $\left(3.7^{\circ}\right.$ vs. $0.34^{\circ}$ (control)), but it cannot be said that this increase was not due to chance alone. The effects of FR on static measures of hip extension, knee flexion, and consequently, rectus femoris length have not yet been investigated. Therefore, the purpose of this study was to investigate the effects of two, one-minute bouts of FR of the anterior thigh on acute hip extension ROM, knee flexion ROM, and rectus femoris length. It was hypothesized that FR of the anterior thigh will acutely increase hip extension ROM, knee flexion ROM, and rectus femoris length. 


\section{Study Design}

This study used a within-subject repeated measures design. Data was collected during one experimental session. Hip extension and knee flexion were measured both before and after one bout of FR, which was carried out by FR the hip flexor muscle group on the anterior thigh. Participants

As per an a priori power analysis $\left(\alpha=0.05 ; \beta=0.80\right.$; expected difference $=2.95^{\circ}$; Cohen's $d=0.54$ ) for an increase in hip extension using $\mathrm{G}^{*}$ Power (Faul et al. 2007), 23 participants $($ male $=7$; female $=16)$ were recruited from a student population via flyers placed on a University campus and presented to Kinesiology and Exercise and Wellness classes. Participants would have been excluded only if they currently had a back or lower extremity musculoskeletal or neuromuscular injury or pain, but no participants reported such an injury. Before each participant was scheduled for testing, the participants were asked about their current injury status. Participants were provided a verbal explanation of the study, and read and signed an Informed Consent and Physical Activity Readiness Questionnaire (PAR-Q) before beginning. Any participant that would have answered "Yes" to any of the questions on the PAR-Q would have been excluded, but none did. Participants' age, height, and weight were then measured. The study was approved by the Institutional Review Board at Arizona State University (IRB ID: STUDY00001660).

\section{Procedures}

A ten minute standardized warm up procedure followed. This warm up consisted of five minutes on an Airdyne bike, two sets of 20 body weight squats, two sets of 10 leg swings in both the frontal and sagittal planes, and two sets of 10 body weight lunges. 

adhered to participants' skin or tight fitting garments on the lateral femoral epicondyle, greater trochanter, lateral malleolus, and iliac crest, halfway between the PSIS and ASIS and spaced $10 \mathrm{~cm}$ apart. These methods differ slightly from those presented by Kuo et al. (2008), as the PSIS and ASIS markers were placed closer to the midaxillary line so they would not be blocked from the camera by the table. Once placed, the markers were not removed until after the final (post-

110 FR) testing procedure. Should the participant's tight fitting garment have had any potential

111 marker distractions (e.g., reflective logos), they were covered with masking tape.

112 Participants then performed one FR intervention, utilizing a 91.44 (L) $\times 15.24$ (D) $\mathrm{cm}$

113 polypropylene foam roller (Perform Better, West Warwick, RI) directed at the right anterior

114 thigh for two, 60 -second bouts. While lying prone, participants were instructed to place their

115 body weight on the foam roller, starting at the proximal aspect of the thigh (just inferior to the

116 ASIS) and rolling down the thigh in a kneading-like fashion, slowly reaching the knee (Figure

117 1). Once the foam roller reached the superior knee, participants were instructed to return the

118 roller to the starting position and continue the sequence for the remainder of the 60 seconds

119 (MacDonald et al. 2013). Participants were instructed to complete the intervention at a slow pace

120 (seconds/repetition). Following a thirty-second break, the participant repeated this intervention.

122 Figure 1 about here.

123

124 Within one minute of completing the second bout of FR, participants' hip extension and

125 knee flexion ROM were re-tested. 
Testing and Analysis

128 Hip extension and knee flexion were measured as the participant performed the modified

129 Thomas test. Hip extension values were calculated by subtracting the four-point angles that the

130 four markers create from $90^{\circ}$. Knee flexion values were calculated by subtracting the three-point

131 angles that the three markers create from $180^{\circ}$. Two-dimensional sagittal plane motion capture

132 were obtained using a $120 \mathrm{~Hz}$ camera, set to $30 \mathrm{~Hz}$ (Basler Scout scA640-120, Basler Vision

133 Technologies, USA), and motion analysis software (MaxTRAQ 2D, Innovision Systems Inc.,

134 USA). Marker digitization was also completed in MaxTRAQ, using auto-digitization and auto-

135 tracking, as to prevent investigator bias. These methods (motion capture) differ substantially

136 from those previously described (Harvey 1998) in that the hip angle was measured relative to the

137 pelvis rather than the plinth (Figure 2). This prevented lumbopelvic movement from confounding

138 the results of the modified Thomas test, which can severely impact the test's reliability (Kim \&

139 Ha 2015). Furthermore, measuring these angles via motion capture presumably allows for more

140 reliable and objective measures, as the same points are being utilized to calculate the angle with

141 each measurement trial. Doing so has demonstrated very high levels of reliability for knee

142 flexion $\left(\mathrm{ICC}=0.98 ; \mathrm{SEM}=1.0^{\circ}\right)($ Peeler \& Leiter 2013) and hip extension $(\mathrm{ICC}=0.90-0.95$;

$143 \mathrm{SEM}=2.0^{\circ}$ ) (Wakefield et al. 2015). The average of three tests was used for each participant's

144 reported measure. Rectus femoris length was estimated using the regression equations and

145 coefficients provided by Hawkins \& Hull (1989), and is presented relative to length at neutral

146 (that is, hip and knee at $0^{\circ}$ ) (Eq. 1); for example, 1.020 would represent a $\%$ increase from

147 resting length, or $102 \%$ of resting length. Similar methods and presentation of data were utilized

148 by Thelen et al. (2004) and Vigotsky et al. (2015). 


$$
l_{R F}=\frac{1.107-\left(1.50 \cdot 10^{-3}\right) \theta_{h i p}+\left(1.99 \cdot 10^{-3}\right) \theta_{\text {knee }}}{1.107}
$$

151

152

153

154

155

156

157

158

159

160

161

163

164

165

166

167

168

169

170

171

172

Figure 2 about here.

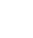

The averages of the three pre- and post-FR measures of hip extension ROM, knee flexion ROM, and calculated rectus femoris length were entered into Stata 13 (StataCorp, LP, College Town, TX). Shapiro-Wilk tests were performed to ensure normality. For normal data, paired samples $t$-tests were performed. Any data found to be non-parametric were compared using Wilcoxon paired-samples signed-rank tests. Alpha was set to 0.05. Parametric effect sizes (ES) were calculated by Cohen's $d$ using the formula $d=\frac{M_{d}}{s_{d}}$, where $M_{d}$ is mean difference and $s_{d}$ is the standard deviation of differences (Becker 1988; Morris 2007; Smith \& Beretvas 2009). This method is slightly different than the traditional method of calculating Cohen's $d$, as it calculates the within-subject effect-size rather than group or between-subject effect sizes. Cohen's $d$ was defined as small, medium, and large for 0.20, 0.50, and 0.80, respectively (Cohen 1988). Nonparametric ES were reported in terms of Pearson's $r$. Pearson's $r$ was defined as small, medium, and large for $0.10,0.30$, and 0.50 , respectively (Cohen 1988). 95\% confidence limits (95\% CL) for effect sizes were also calculated. Because a small number of preplanned comparisons were made, no correction was employed.

\section{Results}

Twenty-three healthy participants (Table 1) were recruited and underwent two, 1-minute bouts of FR the anterior thigh. Measures of hip extension did not meet parametric assumptions, 
173 but knee flexion and rectus femoris length did. Although the increase in hip extension (change $=$

$174+1.86^{\circ}(+0.11,+3.61) ; \mathrm{z}(22)=2.08 ; p=0.0372$; Pearson's $\left.r=0.43(0.02,0.72)\right)$ was not due to

175 chance alone, it cannot be said that the observed changes in knee flexion (change $=-1.39^{\circ}(-5.53$,

$176+2.75) ; \mathrm{t}(22)=-0.70 ; p=0.4933$; Cohen's $d=-0.15(-0.58,0.29))$ or rectus femoris length

$177 \quad$ (change $=-0.005(-0.013,+0.003) ; \mathrm{t}(22)=-1.30 ; p=0.2070 ;$ Cohen's $d=-0.27(-0.70,0.16))$

178 were not due to chance alone (Table 2).

179

180 Table 1 about here.

181

182 Table 2 about here.

183

184 Discussion

185 The purpose of this study was to determine if FR applied to the anterior thigh increases

186 hip extension, knee flexion, and rectus femoris length during the modified Thomas test. It

187 appears that the moderate to large effect observed for hip extension was not due to chance alone.

188 However, it cannot be said for certain that this increase was not at the expense of a decrease in

189 knee flexion, especially since no real change in rectus femoris length was observed. Although

190 prior research would consider the observed increase in hip extension to be clinically relevant - as

191 the observed 45.24\% increase in hip extension exceeds the 10\% threshold (Roach \& Miles 1991)

192 - one cannot truly compute a relative change of joint kinematics, as joint angles are an interval

193 scale, and not ratio scale (O'Donoghue 2014). Additionally, and perhaps more importantly, the

194 increase in hip extension did not exceed the previously-reported SEM of 2.0 (Wakefield et al. 
195 2015), implying that the mean change observed in this trial would not be clinically detectable

196 within or between individuals.

197 Of interest is the inter-individual variability to the FR intervention, as there were

198 responders, non-responders, and even decreases in rectus femoris length observed in individuals

199 (Table 2). For example, participant \#1's rectus femoris length increased by 3.9\%, while

200 participant \#23's rectus femoris length decreased by 3.1\%, and participant \#11 nearly did not

201 experience any change $(-0.1 \%)$. Furthermore, those who had similar changes in rectus femoris

202 length did not necessarily experience those changes from the same place; participant \#22

203 experienced a large increase in hip extension with a decrease in knee flexion, while participant

$204 \# 23$ experienced a small increase in hip extension and a large decrease in knee flexion, but both

205 participants saw similar decreases in rectus femoris length (-0.029 and -0.031 , respectively).

206 Interestingly, there did not appear to be differences in responses between genders (Table 2).

207 These results differ slightly from MacDonald et al. (2013), who found an increase in knee

208 flexion with the hip fixed in extension. However, it should be noted that MacDonald and

209 colleagues' ROM testing utilized a less objective protocol because the participants' passive range

210 of movement was measured while the experimenter actively applied a force to flex their knee.

211 Our protocol involved two 1-minute bouts FR on the anterior thigh, which was identical

212 to the dosage investigated by both MacDonald et al. (2013) and Markovic (2015), who each

213 assessed changes in knee flexion angle following FR of the anterior thigh and reported

214 significant increases. However, it was smaller than the dosage assessed by Bushell et al. (2015),

215 who investigated changes in hip extension angle during a lunge movement following three 1-

216 minute bouts of FR on the anterior thigh and reported significant increases. Given that our

217 protocol involved dosages at the lower end of what has previously been utilized in the literature, 
218 it is possible that it could potentially have been insufficient to bring about changes in flexibility.

219 However, since similar protocols have reported increases in flexibility and since no previous trial

220 has yet identified a dose-response effect following self-manual therapy (Bradbury-Squires et al.

221 2015; Sullivan et al. 2013), this would seem unlikely. Furthermore, several other trials making

222 use of smaller dosages of FR, albeit in other muscle groups, have all reported significant

223 increases in flexibility (Halperin et al. 2014; Škarabot et al. 2015; Sullivan et al. 2013). Together,

224 these factors suggest that the dosage used in our protocol was likely sufficient.

225 The only external force applied to the thigh, resulting in a hip extension moment, was the

226 weight of the participants' lower extremity. Also, since the setup for the modified Thomas test is

227 nearly identical each time, the moment arm about each segment (thigh and leg) is likely similar

228 on each setup. However, it is possible that these moment arms change depending on the

229 compliancy of the rectus femoris. The constant external force and presumably moment arm, and

230 thus external hip extension moment, may provide insight into the mechanisms of self-manual

231 therapy. If FR's effectiveness is a result of an increase in tissue extensibility, an increase in

232 muscle-tendon unit length (with a related decrease in tissue stiffness) with the same applied

233 moment would have been observed due to a shift in the length-tension curve (Weppler \&

234 Magnusson 2010). However, a similar applied moment alone was not enough to elicit observable

235 changes in rectus femoris length. Following these outcomes, it is proposed that self-manual

236 therapy may work through an increase in stretch tolerance rather than an increase in tissue

237 length, as an increase in tissue length or decreased stiffness would have resulted in increased

238 rectus femoris length with the same applied tension. This is certainly possible, as potential

239 mechanisms for manual therapy have been described to be primarily neurophysiological in

240 nature (Bialosky et al. 2009). Recently, Eriksson Crommert et al. (2014) described similar effects 
241 after a seven-minute massage; a decrease in muscle stiffness, measured via elastography, was

242 observed immediately following intervention, but there was no observed effect at three minutes.

243 These findings are similar to ours, in that no changes in stiffness were observed shortly following

244 intervention. This warrants further research utilizing dynamometry, elastography, or similar

245 methods to measure passive joint or muscle stiffness before and following a FR protocol.

246 The mechanism by which FR of the anterior thigh increases flexibility at the hip or knee

247 might inform an understanding of similar interventions intended to reduce rectus femoris strain

248 injury risk. Reviewing rectus femoris strain injury in soccer, Mendiguchia et al. (2012) suggested

249 that the reduced capacity for using the stretch-shortening cycle during the kicking action that

250 might follow from less hip extension ROM, resulting from less hip flexor extensibility, could be

251 key for an increased risk of strain injury. Reduced capacity for using the stretch-shortening cycle

252 could require the rectus femoris to produce more muscle force for each kicking action, thereby

253 increasing the rate of fatigue and consequently the risk of injury. It has been suggested that

254 increasing muscle compliance could reduce muscle strain injury risk in the stretch-shortening

255 cycle in general by enhancing the ability of the muscle-tendon unit to store energy (Witvrouw et

256 al. 2004). Similarly, it has been argued that since strain injuries occur in stretch (Mendiguchia et

257 al. 2012), a stiffer, less flexible muscle might be less likely to incur a strain injury than a

258 compliant one (Gleim \& McHugh 1997; Noonan \& Garrett 1999; Safran et al. 1989). Since the

259 findings of our investigation indicate that FR might exert its effects through an increase in stretch

260 tolerance rather than biomechanical mechanisms (as no change in muscle length was observed

261 and the test did not require additional tension) an increase in flexibility following FR may not

262 provide the purported benefits that could reduce rectus femoris strain injury risk through

263 increases in muscle compliance. 
265 the warm-up participants endured during this study, which was assumed to more closely mimic

266 the warm-up that athletes would typically undergo. The warm-up protocol employed was longer

267 than that of MacDonald et al. (2013), who only had participants perform five minutes on a cycle

268 ergometer. It is possible that the participants in our study had already maximized the potential

269 acute rectus femoris extensibility gains before testing began (O'Sullivan et al. 2009), especially

270 since it been shown that FR and dynamic stretching may elicit similar gains in hip flexion ROM

271 (Behara \& Jacobson 2015). Therefore, the extensive warm-up protocol must be taken into

272 account when interpreting these results. Second, the pace at which participants completed the FR

273 intervention was not recorded, and this might have had an effect on individual outcomes (i.e.,

274 pace-dependent outcomes). Thirdly, the only external force applied to the thigh, resulting in a hip

275 extension moment, was the weight of the participants' lower extremity. Therefore, it is difficult

276 to form conclusions as to whether FR of the anterior thigh would allow a patient or athlete to

277 move through a greater ROM, as the external moment of force during exercise may allow the

278 athlete to increase his or her ROM.

279

280 Conclusions

281 Although a small change in hip extension was observed, no changes in knee flexion or

282 rectus femoris length were observed. From these data, it appears unlikely that FR applied to the

283 anterior thigh will improve passive hip extension and knee flexion ROM, especially if performed

284 in combination with a dynamic stretching protocol. 


\section{References}

Becker BJ. 1988. Synthesizing standardized mean - change measures. British Journal of Mathematical and Statistical Psychology 41:257-278.

Behara B, and Jacobson BH. 2015. The Acute Effects of Deep Tissue Foam Rolling and Dynamic Stretching on Muscular Strength, Power, and Flexibility in Division I Linemen. Journal of Orthopaedic Trauma.

Bialosky JE, Bishop MD, Price DD, Robinson ME, and George SZ. 2009. The mechanisms of manual therapy in the treatment of musculoskeletal pain: a comprehensive model. Manual Therapy 14:531-538.

Bradbury-Squires DJ, Noftall JC, Sullivan KM, Behm DG, Power KE, and Button DC. 2015. Roller-Massager Application to the Quadriceps and Knee-Joint Range of Motion and Neuromuscular Efficiency During a Lunge. Journal of athletic training 50:133-140.

Bushell JE, Dawson SM, and Webster MM. 2015. Clinical Relevance of Foam Rolling on Hip Extension Angle in a Functional Lunge Position. Journal of Strength and Conditioning Research.

Cohen J. 1988. Statistical power analysis for the behavioral sciences: Routledge Academic.

Cross TM, Gibbs N, Houang MT, and Cameron M. 2003. Acute quadriceps muscle strains: magnetic resonance imaging features and prognosis. The American journal of sports medicine 32:710-719.

Eriksson Crommert M, Lacourpaille L, Heales LJ, Tucker K, and Hug F. 2014. Massage induces an immediate, albeit short-term, reduction in muscle stiffness. Scandinavian Journal of Medicine and Science in Sports.

Faul F, Erdfelder E, Lang AG, and Buchner A. 2007. G*Power 3: a flexible statistical power analysis program for the social, behavioral, and biomedical sciences. Behavior Research Methods 39:175-191.

Gleim GW, and McHugh MP. 1997. Flexibility and its effects on sports injury and performance. Sports Medicine 24:289-299.

Halperin I, Aboodarda SJ, Button DC, Andersen LL, and Behm DG. 2014. Roller massager improves range of motion of plantar flexor muscles without subsequent decreases in force parameters. International Journal of Sports Physical Therapy 9:92-102.

Hartmann H, Wirth K, Klusemann M, Dalic J, Matuschek C, and Schmidtbleicher D. 2012. Influence of squatting depth on jumping performance. Journal of Strength and Conditioning Research 26:3243-3261.

Harvey D. 1998. Assessment of the flexibility of elite athletes using the modified Thomas test. British Journal of Sports Medicine 32:68-70.

Hawkins D, and Hull ML. 1989. A method for determining lower extremity muscle-tendon lengths during flexion/extension movements. Journal of Biomechanics 23:487-494.

Healey KC, Hatfield DL, Blanpied P, Dorfman LR, and Riebe D. 2013. The Effects of Myofascial Release with Foam Rolling on Performance. Journal of Strength and Conditioning Research.

Jay K, Sundstrup E, Sondergaard SD, Behm D, Brandt M, Saervoll CA, Jakobsen MD, and Andersen LL. 2014. Specific and cross over effects of massage for muscle soreness: randomized controlled trial. International Journal of Sports Physical Therapy 9:8291. 
Kim GM, and Ha SM. 2015. Reliability of the modified Thomas test using a lumbo-plevic stabilization. Journal of Physical Therapy Science 27:447-449.

Kuo Y-LL, Tully EA, and Galea MP. 2008. Skin movement errors in measurement of sagittal lumbar and hip angles in young and elderly subjects. Gait and Posture 27:264-270.

Macdonald GZ, Button DC, Drinkwater EJ, and Behm DG. 2014. Foam rolling as a recovery tool after an intense bout of physical activity. Medicine and Science in Sports and Exercise 46:131-142.

MacDonald GZ, Penney MD, Mullaley ME, Cuconato AL, Drake CD, Behm DG, and Button DC. 2013. An acute bout of self-myofascial release increases range of motion without a subsequent decrease in muscle activation or force. Journal of Strength and Conditioning Research 27:812-821.

Markovic G. 2015. Acute effects of instrument assisted soft tissue mobilization vs. foam rolling on knee and hip range of motion in soccer players. Journal of Bodywork and Movement Therapies.

McMahon GE, Morse CI, Burden A, Winwood K, and Onambele GL. 2014. Impact of range of motion during ecologically valid resistance training protocols on muscle size, subcutaneous fat, and strength. Journal of Strength and Conditioning Research 28:245-255.

Mendiguchia J, Alentorn-Geli E, Idoate F, and Myer GD. 2012. Rectus femoris muscle injuries in football: a clinically relevant review of mechanisms of injury, risk factors and preventive strategies. British Journal of Sports Medicine.

Morris SB. 2007. Estimating effect sizes from the pretest-posttest-control group designs. Organizational Research Methods.

Morton SK, Whitehead JR, Brinkert RH, and Caine DJ. 2011. Resistance training vs. static stretching: effects on flexibility and strength. Journal of Strength and Conditioning Research 25:3391-3398.

Noonan TJ, and Garrett WE, Jr. 1999. Muscle strain injury: diagnosis and treatment. Journal of the American Academy of Orthopaedic Surgeons 7:262-269.

O'Donoghue P. 2014. An Introduction to Performance Analysis of Sport: Routledge.

O'Sullivan K, Murray E, and Sainsbury D. 2009. The effect of warm-up, static stretching and dynamic stretching on hamstring flexibility in previously injured subjects. $B M C$ Musculoskeletal Disorders 10:37.

Okamoto T, Masuhara M, and Ikuta K. 2013. Acute Effects of Self-Myofascial Release Using a Foam Roller on Arterial Function. Journal of Strength and Conditioning Research.

Orchard J, and Seward H. 2002. Epidemiology of injuries in the Australian Football League, seasons 1997-2000. British Journal of Sports Medicine 36:39-44.

Pearcey GE, Bradbury-Squires DJ, Kawamoto JE, Drinkwater EJ, Behm DG, and Button DC. 2014. Foam Rolling for Delayed-Onset Muscle Soreness and Recovery of Dynamic Performance Measures. Journal of Athletic Training.

Peeler J, and Leiter J. 2013. Using digital photography to document rectus femoris flexibility: A reliability study of the modified Thomas test. Physiotherapy Theory and Practice 29:319-327.

Roach KE, and Miles TP. 1991. Normal hip and knee active range of motion: the relationship to age. Physical Therapy 71:656-665.

Safran MR, Seaber AV, and Garrett WE, Jr. 1989. Warm-up and muscular injury prevention. An update. Sports Medicine 8:239-249. 
386

387

Schroeder AN, and Best TM. 2015. Is self myofascial release an effective preexercise and recovery strategy? A literature review. Current Sports Medicine Reports 14:200-208. Škarabot J, Beardsley C, and Stirn I. 2015. Comparing the effects of self-myofascial release with static stretching on ankle range-of-motion in adolescent athletes. International Journal of Sports Physical Therapy 10:203-212.

Smith LJW, and Beretvas SN. 2009. Estimation of the Standardized Mean Difference for Repeated Measures Designs. Journal of Modern Applied Statistical Methods 8:27. Speer KP, Lohnes J, and Garrett WE. 1992. Radiographic imaging of muscle strain injury. The American journal of sports medicine 21:89.

Sullivan KM, Silvey DB, Button DC, and Behm DG. 2013. Roller-massager application to the hamstrings increases sit-and-reach range of motion within five to ten seconds without performance impairments. International Journal of Sports Physical Therapy 8:228-236.

Thelen DG, Chumanov ES, Hoerth DM, Best TM, Swanson SC, Li L, Young M, and Heiderscheit BC. 2004. Hamstring muscle kinematics during treadmill sprinting. Medicine and Science in Sports and Exercise 37:108-114.

Vigotsky AD, Harper EN, Ryan DR, and Contreras B. 2015. Effects of load on good morning kinematics and EMG activity. PeerJ 3:e708.

Wakefield CB, Halls A, Difilippo N, and Cottrell GT. 2015. Reliability of goniometric and trigonometric techniques for measuring hip-extension range of motion using the modified Thomas test. Journal of Athletic Training 50:460-466.

Weppler CH, and Magnusson SP. 2010. Increasing muscle extensibility: a matter of increasing length or modifying sensation? Physical Therapy 90:438-449.

Witvrouw E, Mahieu N, Danneels L, and McNair P. 2004. Stretching and injury prevention. Sports medicine 34:443-449.

Wyon MA, Smith A, and Koutedakis Y. 2013. A comparison of strength and stretch interventions on active and passive ranges of movement in dancers: a randomized controlled trial. Journal of Strength and Conditioning Research 27:3053-3059. 
1

Starting (A) and ending (B) position of the foam roll protocol.
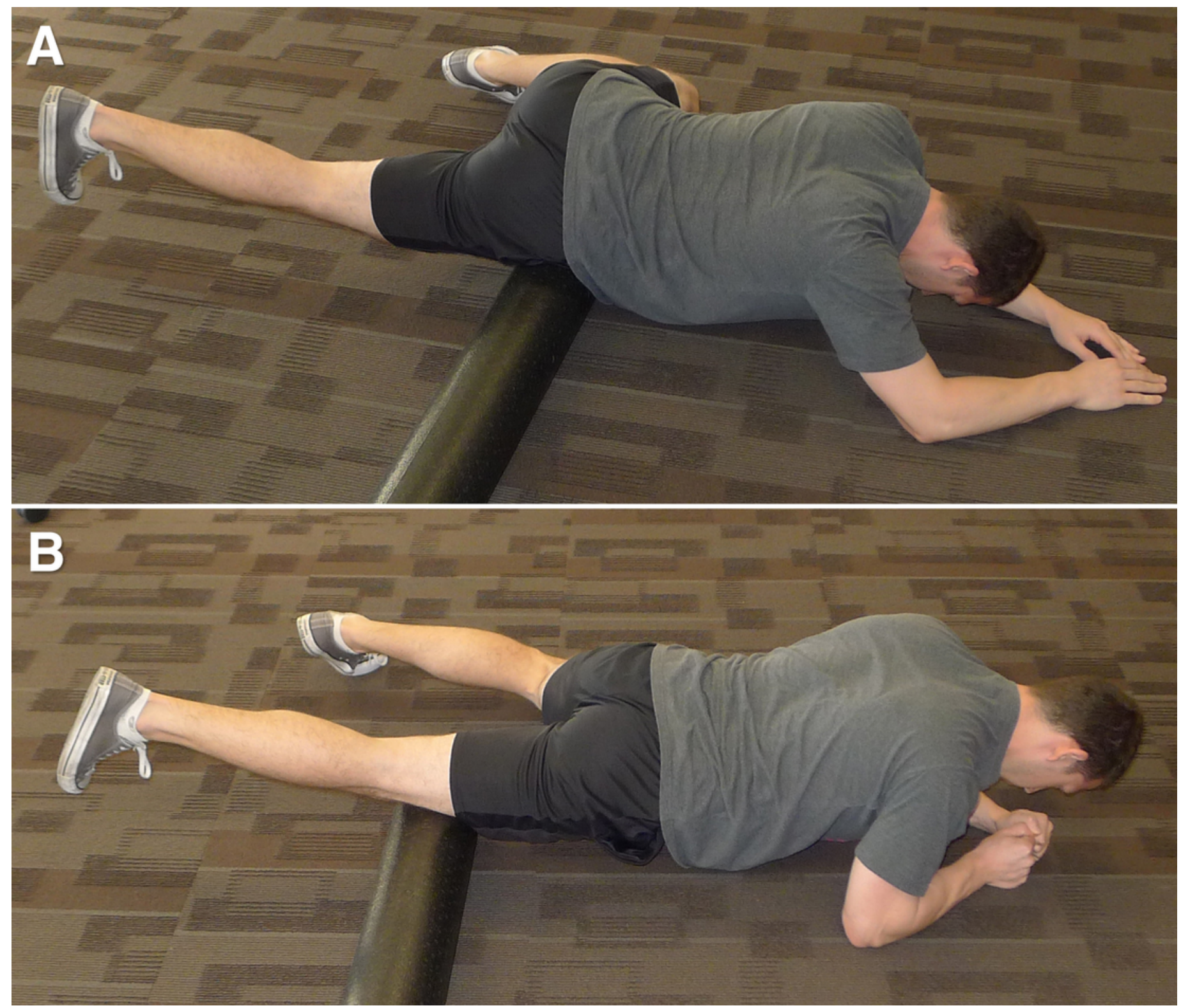
Figure 2 (on next page)

Hip and knee joint calculations.

The illustrated participant would have a hip extension angle of $8.1^{\circ}\left(98.1^{\circ}-90^{\circ}\right)$ and a knee flexion angle of $53.2^{\circ}\left(180^{\circ}-126.8^{\circ}\right)$. Illustration credit: Ji Sung Kim 


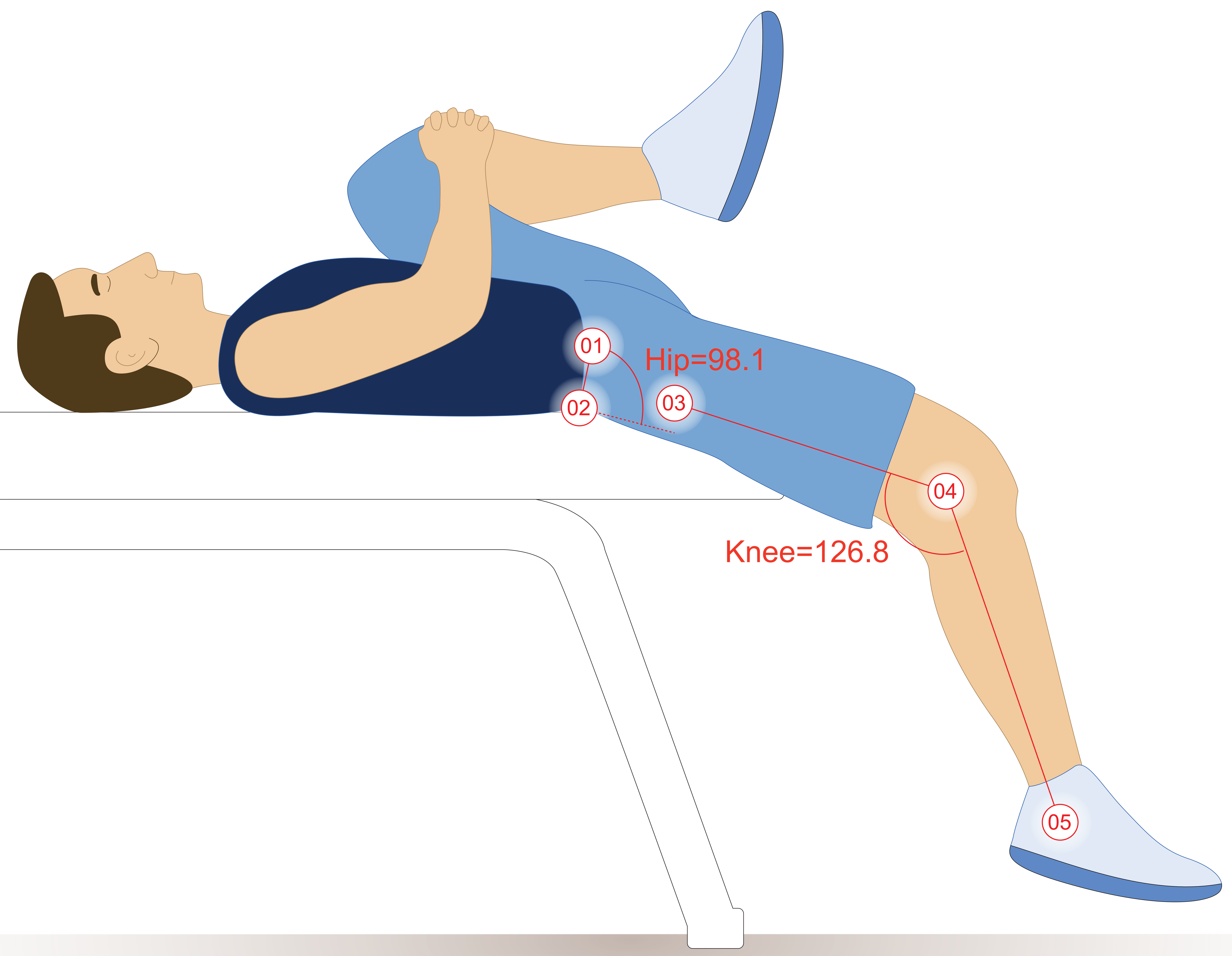




\section{Table $\mathbf{1}$ (on next page) \\ Descriptive statistics of participants.}


1

2 Table 1. Descriptive statistics of participants

\begin{tabular}{|l|l|l|l|l|}
\hline Sex & n & Age (years) & Height $(\mathbf{c m})$ & Body mass (kg) \\
\hline Male & 7 & $21.00 \pm 1.63$ & $179.54 \pm 6.90$ & $83.24 \pm 11.25$ \\
\hline Female & 16 & $22.06 \pm 3.84$ & $165.74 \pm 6.53$ & $60.91 \pm 10.64$ \\
\hline Total & 23 & $22.00 \pm 3.30$ & $169.95 \pm 9.18$ & $67.71 \pm 14.90$ \\
\hline
\end{tabular}

3 
Table 2 (on next page)

Individual and mean ( $\pm \mathrm{SD}$ ) changes in hip extension ROM, knee flexion ROM, and calculated rectus femoris length pre- and post-FR. 
1 Table 2. Individual and mean ( \pm SD) changes in hip extension, knee flexion, and calculated rectus femoris length pre- and post-FR.

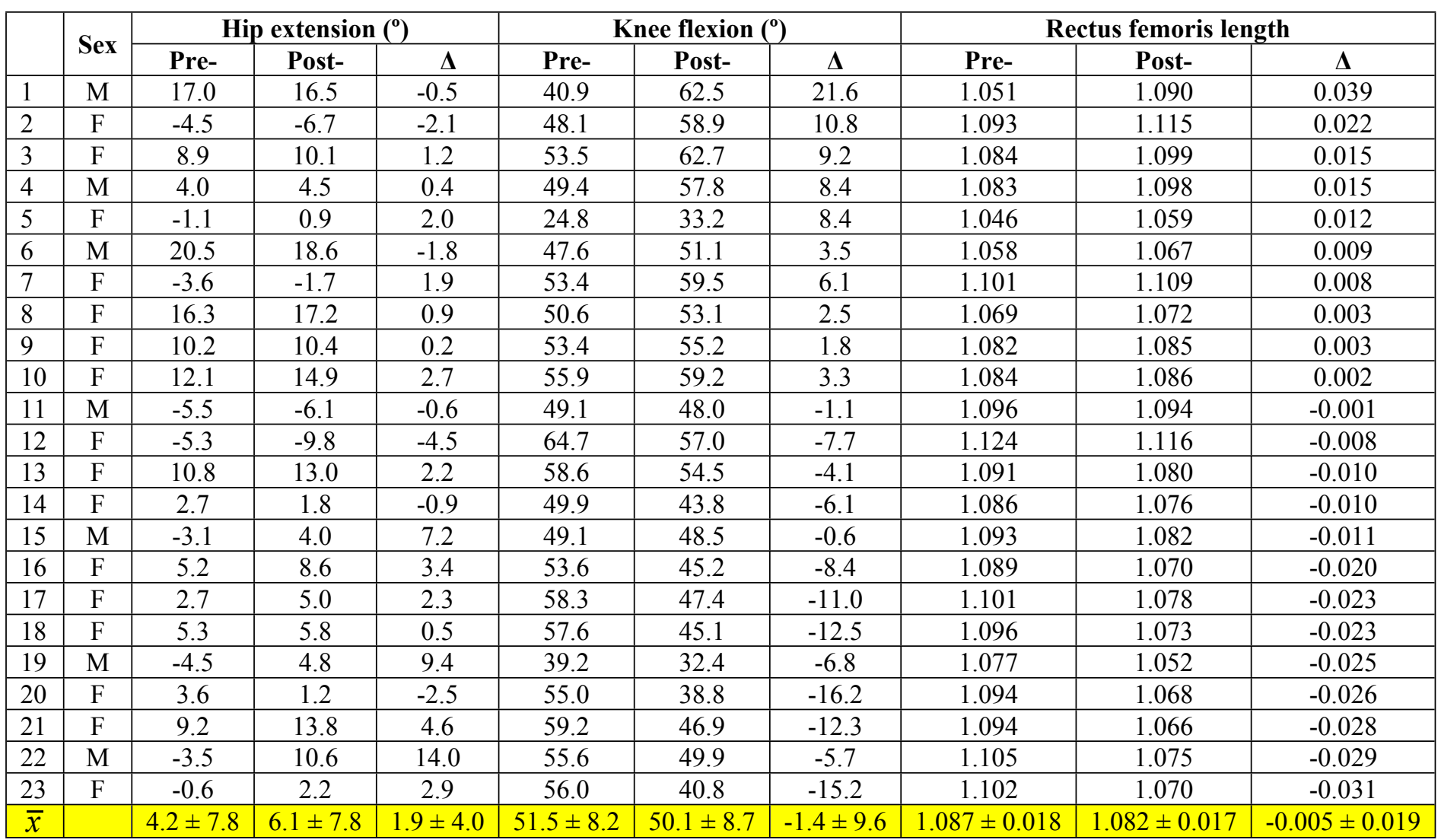

